\title{
Marshall y los sistemas productivos locales
}

Marshall and local production systems

\author{
Jorge Carreto Sanginés
}

\section{Resumen}

La eliminación de barreras comerciales y la integración de mercados nacionales individuales en áreas de libre comercio han permitido un enorme aumento en el comercio y las transacciones financieras internacionales. Este desarrollo ha dado lugar a nuevas tendencias de aglomeración de la actividad económica, diversas en cuanto a las reglas de la competencia, a la colaboración en la producción y a las modalidades de generación e intercambio de conocimientos, en las que prevalecen nuevas formas de organización productiva a nivel local, con una tendencia a aglutinar empresas y a integrar sistemas productivos locales (SPL). Los sistemas productivos locales han adquirido importancia por su papel en la generación de innovaciones y de nuevo conocimiento. Los llamados "distritos marshallianos", son organizaciones productivas localizadas geográficamente y con autonomía en la generación y aplicación de nuevas tecnologías, que parten del aprovechamiento de conocimientos locales y, en ocasiones, de carácter tradicional. Alfred Marshall, a finales del siglo XIX, notó cómo la presencia conjunta de empresas de un mismo sector y en la misma área creara una "atmósfera industrial" de "mutua confianza y conocimiento" capaz de sostener e impulsar a la industria local. Es en este contexto en el que algunos de los conceptos marshallianos de la competencia, cobran nuevo interés en el análisis de los sistemas productivos locales, en particular, sus nociones sobre la difusión del conocimiento y las economías externas. En particular, permiten indagar sobre las causas que llevan a las empresas a aglomerarse en distritos industriales y se pueden encontrar conceptos enriquecedores para el análisis y lineamientos para definir estrategias de desarrollo regional.

\section{Palabras clave:}

- Microeconomía

- Economía espacial general

- Comportamiento de las economias domésticas

JEL: B21, R1, D1

\section{Abstract}

The reductions in trade barriers and the integration of national markets in free trade zones have produced an enormous increase in trade and international financial transactions. This development has set the grounds for new trends in the agglomeration of economic activity, differing in the rules of competition and in the modes of productive collaboration, as well as the in way knowledge is generated and exchanged, and in which new forms of productive organization prevail at a local level, with a tendency to attract new firms and to create local productive systems (LPS). These LPS have gained importance due to their role in the generation of innovations and new knowledge. The so-called "marshallian districts" are productive organisations located geographically and with autonomy in the generation and application of new technologies, which flourish upon local knowledge, sometimes of a traditional character. Alfred Marshall, at the end of XIX century noted that the presence of several firms belonging to the same sector and the same area helped creating an "industrial atmosphere" of "mutual confidence and acquaintance" able to sustain and promote local industrial development. It is in this context that some of the marshallian concepts of competition acquire new interest in the analysis of LPS, and particularly, the notions about the diffusion of knowledge and external economies. In particular, they allow to explore the causes behind the agglomeration of firms in industrial districts and to find concepts that might be useful in the analysis and definition of regional development strategies.

Keywords:

- Microeconomics

- General Spatial Economics

- Household Behavior

\section{Introducción}

La apertura comercial y la fusión de mercados nacionales individuales en áreas de libre comercio se han acompañado de un aumento en el comercio y las transacciones financieras internacionales. Estas condiciones están redefiniendo las reglas de la competencia y colaboración productivas, así como de ge- 
neración e intercambio de conocimientos. En este escenario, están surgiendo nuevas formas de organización productiva basadas en la cohabitación competitiva y colaborativa a nivel local y territorial, que tienden a conformar aglomeraciones entre empresas y a integrar sistemas productivos locales (SPL).

Los sistemas productivos locales han adquirido importancia por su papel en la generación de innovaciones y de nuevo conocimiento. Los llamados “distritos marshallianos", son organizaciones productivas localizadas geográficamente y con autonomía en la generación y aplicación de nuevas tecnologías, que parten del aprovechamiento de conocimientos locales y, en ocasiones, de carácter tradicional.

Alfred Marshall, a finales del siglo xix, notó cómo la presencia conjunta de empresas de un mismo sector y en la misma área creara una "atmósfera industrial" de "mutua confianza y conocimiento" capaz de sostener e impulsar a la industria local. Es en este contexto en el que algunos de los conceptos marshallianos de la competencia, cobran nuevo interés en el análisis de los sistemas productivos locales, en particular, sus nociones sobre la difusión del conocimiento y las economías externas.

En particular, permiten indagar sobre las causas que llevan a las empresas a aglomerarse en distritos industriales y se pueden encontrar conceptos enriquecedores para el análisis y lineamientos para definir estrategias de desarrollo regional.

\section{Sistemas productivos locales}

La reducción de barreras nacionales al comercio y a las transacciones financieras, y la fusión de mercados nacionales individuales en áreas de libre mercado, han generado un enorme aumento del comercio internacional y de los flujos de capital entre diferentes países. Las condiciones que caracterizan a la actual "sociedad del conocimiento", están redefiniendo la competencia en los mercados y la generación e intercambio de conocimientos en todos los ámbitos. En este escenario han surgido modalidades de organización productiva alternativas basadas en la cohabitación competitiva y colaborativa a nivel local y territorial, que tienden a conformar aglomeraciones entre diversos productores y a integrar sistemas productivos locales (SPL).

La importancia de estas modalidades organizacionales, dada su capacidad de adaptación al cambio y su creatividad, ha dado lugar a un gran interés por

${ }^{1}$ De acuerdo con el concepto empleado por la unESCO en su informe: "Hacia las sociedades del conocimiento" (UNESCO, 2005). 
Economía Informa núm. 383 noviembre - diciembre • 2013 | " " "

abordar el estudio del desarrollo industrial desde un enfoque local. El tema ha atraído la atención desde hace ya varias décadas, por lo que abunda la literatura que analiza una gran cantidad de casos de clusters y SPL — una serie de "densos nodos de trabajo humano y vida comunitaria, desperdigados por el mundo", formaciones sociales subnacionales (regionales) distintivas en donde se realizan actividades productivas de todo tipo; (Scott, 2001) - que se localizan en regiones y países con diferentes niveles de desarrollo.

Existe una visión muy difundida que considera que las pequeñas y medianas empresas se encuentran generalmente en desventaja en la competencia global frente a las grandes compañías multinacionales (CMN) que ocupan todos los espacios del mercado. ${ }^{2}$ La liberalización de movimientos de capitales y la desregulación financiera a nivel global han dado lugar a un enorme crecimiento de las inversiones extranjeras directas, en la mayor parte de los casos reubicando procesos productivos hacia países que ofrecen algún tipo de ventaja comparativa. Las grandes CMN que realizan inversiones a gran escala en diferentes países tienen la capacidad de transformar la estructura productiva de las economías regionales en las que se instalan y desplazar a las industrias tradicionales. Sin embargo, a partir de la crisis del modelo industrial fordista, han surgido fórmulas de organización de la producción alternativas a las estructuras integradas verticalmente y bajo el control financiero, comercial y tecnológico de las grandes corporaciones. Existe evidencia que muestra que pequeñas empresas organizadas localmente pueden sobreponerse a restricciones como el difícil acceso a tecnología, a insumos, a mercados, información y conocimiento, crédito y servicios externos, y que "en las últimas décadas, la innovación se crea cada vez más y en mayor medida en forma de vinculaciones o redes, eso es, en un entorno en el que la empresa es uno más entre diversos actores que la impulsan, crean o transfieren..." (Jasso, 2004, p. 122).

A partir de esto, surge el interés por recuperar algunos conceptos analíticos que describan los elementos, las estructuras y los mecanismos de funcionamiento de los sistemas productivos locales en general y permitan identificar planteamientos estratégicos originales. La teoría económica encuentra dificultades para incorporar el cambio técnico y el conocimiento en sus modelos y para explicar los fenómenos sociales que acompañan al funcionamiento de las empresas dentro de las redes, particularmente en relación con la generación y difusión de conocimientos e ideas nuevos.

\footnotetext{
${ }^{2}$ La idea de que las pequeñas y medianas empresas se encuentran en desventaja es resumida por el Principio de Asimetría de Joseph Steindl, que establece que todo lo que puede ser hecho por una pequeña empresa, puede ser hecho por una gran empresa, pero no a la inversa.
} 
La tipología de sistemas productivos y redes de empresas es muy variada. Michael Porter (1998) define a los clusters como concentraciones geográficas de empresas interconectadas, proveedores especializados, proveedores de servicios, empresas de industrias relacionadas e instituciones asociadas, como universidades, agencias certificadoras y asociaciones comerciales. El concepto crucial, de acuerdo con Porter, es que operan en campos específicos y compiten entre sí, pero a la vez cooperan entre sí. Sin embargo, para Porter los nuevos inventos, perfeccionamientos en las tecnologías básicas, acontecimientos políticos externos y cambios sustanciales en la demanda de los mercados extranjeros son variables casuales que suceden fuera del control de las empresas y los "recursos de conocimiento" estarían constituidos por la dotación que la nación tenga de conocimientos científicos, técnicos y de mercado que importen para los bienes y los servicios, y las fuentes de estos conocimientos se encontrarán en las universidades, los organismos estatales de investigación; las entidades privadas de investigación (Porter, 1990, p. 115). Existen en los SPL, sin embargo, externalidades que son totalmente diferentes de las ventajas comparativas ("efectos de país") generadas por el atractivo del ambiente general en el cual la compañía está ubicada, y conocimientos que son generados y reproducidos al interior del SPL.

Otras clasificaciones propuestas distinguen a los SPL a partir de criterios como las dimensiones de las empresas, las relaciones entre las mismas, su orientación tanto hacia el interior como hacia el exterior de la red, el tipo de tecnología, etc. Una clasificación sencilla, basada en las relaciones con la red, distingue entre distrito industrial marshalliano, distritos hub-and-spoke ${ }^{3}$, y plataformas satelitales ${ }^{4}$ (Markusen, 1996). Otra tipología distingue entre distritos tecnológicos, clusters regionales o milieux, y distritos industriales estándar (Camisón, 2003).

En el presente trabajo nos referiremos a los distritos industriales marshallianos o estándar. Se trata de redes compuestas por empresas pequeñas y medias especializadas sectorialmente, que establecen relaciones de "co-ompetencia" (cooperación y competencia simultánea) basadas en relaciones de confianza, coordinadas a través del involucramiento de instituciones locales. La mayor parte están integrados por industrias pertenecientes a sectores maduros, de tipo industrial o artesanal. El ejemplo más conocido es el de los distritos industriales italianos, que se han desarrollado a partir de la década de 1970

\footnotetext{
${ }^{3}$ Distritos hub-and-spoke. Se trata de redes que forman un perno sobre una o más empresas de grandes dimensiones, ligadas con otras empresas o subsidiarias que operan fuera de la dimensión local. ${ }^{4}$ Plataforma satelital. Redes formadas por las subsidiarias de empresas más amplias, que concentran parte de la propia producción en un área determinada.
} 
Economía Informa núm. 383 noviembre - diciembre • 2013 | " " "

en diferentes ramas industriales caracterizándose por la existencia de sistemas de pequeñas y medianas empresas con gran capacidad para la exportación y de adaptación a las crisis económicas. Estas empresas se localizan en áreas limitadas territorialmente, particularmente en algunas ciudades pequeñas o de tamaño medio en el centro y noreste de Italia (como Prato, Módena o Carpi).

Los recientes análisis sobre sPLs industriales, en particular los distritos industriales italianos, tuvieron la importante consecuencia de que se discutieran dos temas de gran interés: por un lado, las posibilidades y ventajas competitivas de formas novedosas de organización económica basadas sobre redes de colaboración interempresarial, y la importancia de aspectos socioculturales sobre el desempeño económico, tales como la confianza, la reciprocidad, las pautas de comportamiento común, los conflictos locales, los valores compartidos y sus consecuentes sanciones sociales. ${ }^{5}$

\section{La atmósfera industrial}

No es nuestra intención hacer una exposición de la teoría de Alfred Marshall, ni indagar en qué medida su teoría de la empresa haya sido condicionada por la necesidad de incorporar el papel que debe cumplir un modelo de empresa en el contexto del equilibrio económico general y de la teoría de la distribución neoclásica. A lo más, nos aventuraremos a suponer que tal condicionamiento constituía un obstáculo para la libre utilización de la abundante información empírica contenida en su obra. En una recuperación de los conceptos marshallianos es necesario tener presente una fuerte tensión entre dos propósitos fundamentales de la teoría. Por una parte, un requisito de realismo y eficacia interpretativa que obliga a Marshall a reconocer formas competitivas diferentes de la competencia perfecta y, por la otra, la condición - impuesta por la ortodoxia del equilibrio general - de coherencia teórica, y que requiere de una teoría de la empresa que pueda ser insertada sin contradicciones en la teoría del equilibrio económico ${ }^{6}$. Se basó más en argumentos prácticos e intuitivos que en el formalismo matemático de la escuela del equilibrio general;

\footnotetext{
${ }^{5}$ En esta dirección están también otros trabajos sobre la experiencia mexicana como los de Cimoli (coord) $l$ (2000), Casas et al (2001), Jasso y Torres (2008), Corona et al (1995), Corona (2001), Paunero y Corona (2007), Solleiro (2006), Unger (2008), Sánchez Daza et al (2005), Dutrenit (2009) y Rozga (2009).

${ }^{6}$ Sraffa hace alusión a este conflicto en su famoso artículo de 1926: "I am trying to find what are the assumptions implicit in Marshall's theory... We seem to be agreed that the theory cannot be interpreted in a way which makes it logically self-consistent and, at the same time, reconciles it with the facts it sets out to explain. Mr. Robertson's remedy is to discard mathematics, and he suggests that my method is to discard the facts; perhaps I ought to have explained that, in the circumstances, 
trató de incorporar el tiempo, además de la estructura institucional e industrial y los fenómenos del mundo real, tales como la incertidumbre, el dinero y los ciclos financieros.

En la búsqueda de realismo, Marshall se convierte en un heterodoxo. Su concepción biológica del equilibrio de largo plazo antecede al evolucionismo moderno, pero contrasta profundamente con una concepción mecánica del equilibrio económico general. En su teoría de la empresa transcurre un tiempo real, histórico, a diferencia del tiempo virtual del equilibrio walrasiano. En este tiempo histórico en el que las empresas nacen, alcanzan su plenitud y después decaen y mueren, cambian los datos del problema - las funciones de costo- y las funciones de oferta se vuelven irreversibles.

La competencia perfecta de Marshall realmente no era tan perfecta, en virtud de los "mercados especiales", o de las empresas que cuentan con el suficiente poder para impedir que los precios bajen por temor a arruinar los mercados y despertar el odio de los demás competidores (Marshall, 1890). La preocupación por la coherencia con la teoría general no es algo que preocupara demasiado a Marshall, y es precisamente de su afán empírico, en contraste con los principios del equilibrio general, de donde surgen los conceptos de mayor valor heurístico.

$\mathrm{Su}$ principal objetivo consistió en representar las condiciones reales de la economía, más que las condiciones idealizadas de los teóricos continentales del equilibrio general. Partidario de una mayor flexibilidad en el análisis, resumía su pragmatismo: "Todos los instrumentos para el descubrimiento de las relaciones entre causa y efecto que están descritas en los tratados del método científico, deben ser utilizados por el economista: no hay ningún método de investigación que pueda ser propiamente llamado el método de la economía; sino que cada método debe ser utilizable en la ocasión adecuada, ya sea sólo o en combinación con otros" (Principles, Libro I, cap. III). En este tenor, utilizó el "análisis parcial" y justificó su teoría argumentando que todas las doctrinas científicas tácita o implícitamente suponen ciertas condiciones y que las leyes de las "económicas" son "hipotéticas", es decir, realiza el estudio de los efectos que serán producidos por ciertas causas, no de manera absoluta, sino bajo la condición de que las demás causas permanezcan iguales, y que dichas causas puedan surtir sus efectos sin que nada las perturbe ${ }^{7}$ (Principles, Libro I, cap.III).

\footnotetext{
7 It is sometimes said that the laws of economics are "hypothetical." Of course, like every other science, it undertakes to study the effects which will be produced by certain causes, not absolutely, but subject to the condition that other things are equal, and that the causes are able to work out their effects undisturbed.
} 
Economía Informa núm. 383 noviembre - diciembre • 2013 | " " "

En Industry and Trade, Marshall describió cómo la presencia conjunta de empresas en el mismo sector y en la misma área creara una "atmósfera industrial" de "mutua confianza y conocimiento" capaz de sostener e impulsar a la industria local (Marshall, 1919). En esta "atmósfera industrial” de los conglomerados industriales, los costos de transacción se reducen por varias razones: la difusión de códigos comunes, la rápida difusión de la información del sector, la confianza que proporciona el respeto a contratos y acuerdos a través de la reputación.

El concepto de "atmósfera industrial" ha sido criticado por considerársele vago, más bien metafórico; con el desarrollo del concepto de conocimiento tácito se ha podido apreciar el valor económico de la transmisión en persona del know-how para la producción, así como el valor de instituciones informales. La libre circulación de la información y la reducción de los costos de transacción por la confianza entre los miembros del distrito implican un apego a la comunidad, un sentido de pertenencia. En tanto que la teoría económica neoclásica está basada en la representación de individuos y unidades productivas que buscan maximizar una función objetivo, en el análisis de Marshall, el concepto de "sentido de pertenencia" permite ubicar el análisis a un nivel meso, entre lo micro y lo macro. De cualquier manera, los conceptos de "atmósfera industrial" y "sentido de pertenencia" abren el argumento al intercambio entre la economía política, la sociología, la antropología, el urbanismo y la psicología social, dándole al análisis un carácter multidisciplinario.

En el caso de los distritos industriales italianos, la red de relaciones entre las empresas al interior del distrito es más rica que en otros sistemas productivos menos desarrollados y basados en el territorio o en sistemas productivos con carácter jerárquico, dominados por las decisiones de pocas empresas grandes. La interacción entre las empresas permite difundir en el tejido social (y, en primer lugar, entre los empresarios) informaciones sobre las tecnologías, los materiales, los productos: produce, en otras palabras, informaciones económicamente relevantes sobre posibles oportunidades de beneficio y la información que deriva de la experiencia directa de otros empresarios se transmite más rápidamente en el distrito industrial que en otros modelos de manufactura, induciendo un efecto de verdadero "contagio informativo" (Brusco, Minerva, Poli, Solinas, julio, 2001; Grandinetti e Tabacco, 2003; Camisón, 2003). El distrito industrial en este contexto, consiste en un espacio externo de recursos y capacidades — competencias,${ }^{8}$ capital intelectual, conocimien-

\footnotetext{
${ }^{8}$ Entendemos por competencias a esquemas complejos que articulan habilidades, actitudes y conocimientos que las personas aplican para relacionarse con los demás, realizar algún trabajo o actividad, resolver un problema o intervenir en su entorno.
} 
to técnico, conocimiento organizacional - al cual las compañías miembros tienen acceso. Se distingue por su capacidad de generar una amplia variedad productiva fundada principalmente en un proceso de innovación que no surge de centros formales de investigación y desarrollo especializados, sino que tiene lugar de manera distribuida a su interior en un espacio común y en donde la fábrica se convierte en el laboratorio en el que en muchas ocasiones se encuentran las soluciones a los problemas que la empresa enfrenta (learning by doing) (Chiarvesio, et. al, 2003). En la figura 1 se ilustran los factores que se conjuntan en el ámbito del distrito industrial dando lugar a amplias economías externas y ventajas competitivas.

\section{Figura I \\ Esquema del distrito industrial marshalliano}

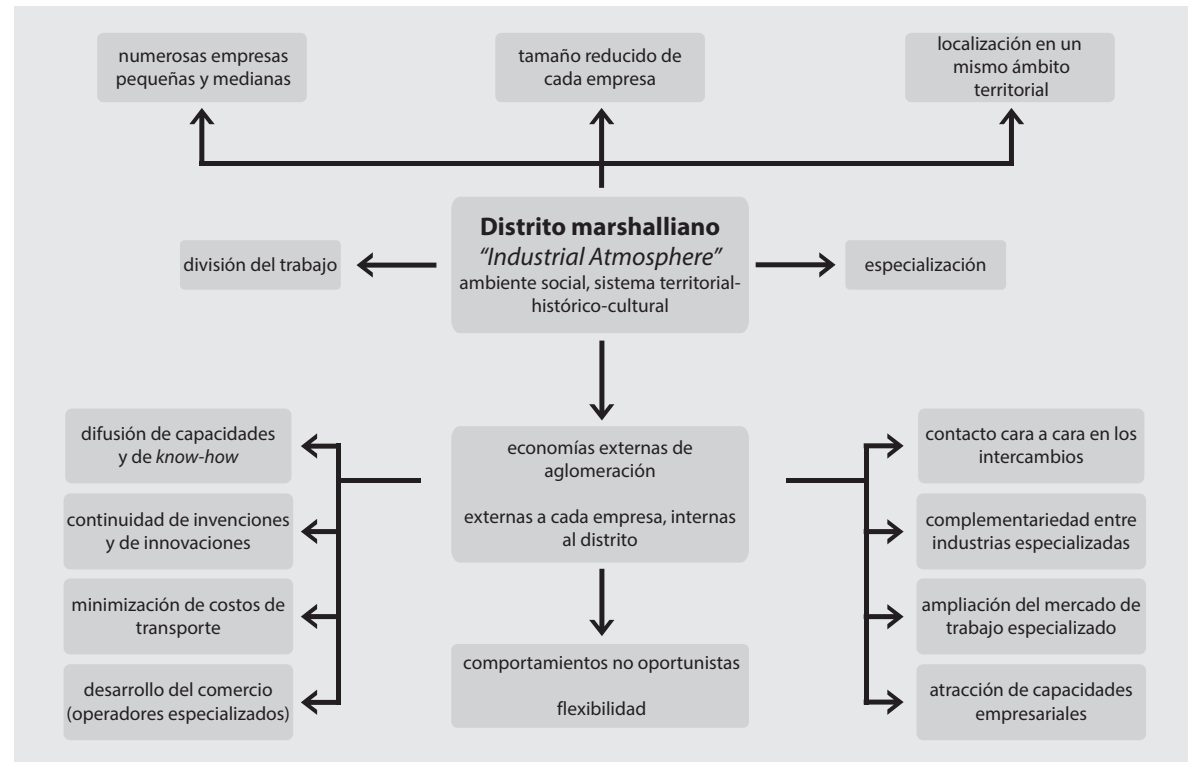

Una forma de abordar el problema de los distritos industriales desde una perspectiva teórica consiste en hacer un análisis estratégico de los mismos considerándolos "comunidades cognitivas", de manera que se cuente con una guía de la investigación dentro de las diferentes redes inter e intra compañías, así como a las fuentes de éxito en los negocios y a la posible contribución de los conglomerados territoriales a este.

El conocimiento, en opinión de Marshall, es la más poderosa máquina de la producción, ya que nos permite "dominar a la naturaleza y forzarla a 
Economía Informa núm. 383 noviembre - diciembre • 2013 | " " "

satisfacer nuestras necesidades" y destacó la importancia de su transmisión: "Los misterios del oficio dejan de ser misterios; como si estuvieran en el aire y los niños aprendiesen de ellos inconscientemente" (Marshall, 1890, iv.i). Las invenciones y mejoras en la maquinaria, en los procesos y, en general, en la organización de los negocios, se difunden, y cuando alguien comienza con una nueva idea, esta es rápidamente adoptada por los demás y combinada con sugerencias propias, con lo cual surgen nuevas ideas (Marshall, 1890 iv.x.7). La organización industrial constituye un cuarto factor productivo, después de trabajo, capital y tierra. En este tenor, el capital consistiría, en su mayor parte, en conocimiento y organización y de este, una parte sería privado y otra no.

\section{Economías externas}

La atmósfera industrial al interior de un distrito industrial depende de la densidad de la red de cooperación y del grado de integración entre las empresas y el contexto social. La ventaja para las empresas que habitan el distrito estriba en el acceso a las competencias compartidas, es decir, el conocimiento tácito que existe en el ambiente interno y al que las organizaciones tienen acceso colectivamente a través de un conjunto de instituciones y reglas que se diseminan dentro del distrito y se transmiten de una generación a otra. Se trata, fundamentalmente, de redes de competencias como las redes de información; redes de interpretación; redes de producción, mediante las cuales colaboran con otros agentes del distrito para crear nuevos tipos de bienes y producir bienes para el mercado, y redes de marketing.

Algunos estudiosos (Camisón, 2003) sugieren adoptar una "Visión Basada en las Competencias", para estudiar la relación causal entre la localización en clusters y el desempeño organizacional (medido como un valor de mercado). Mediante este enfoque es posible ponderar la contribución al desempeño de la empresa, del conjunto de recursos y capacidades de las compañías individualmente y el de las competencias compartidas en el distrito. ${ }^{9}$ En la esfera compartida o colectiva del distrito industrial, las competencias consisten en conocimiento tácito e idiosincrasias propias del ambiente interno del distrito y a las que las organizaciones ubicadas a su interior tienen acceso colectivamente" (Camisón, 2003). Estas competencias son, principalmente, la adquisición de conocimiento intra distrito, el aprendizaje colectivo, una visión compartida,

\footnotetext{
${ }^{9}$ Entendemos por competencias a estructuras cognitivas complejas, que articulan habilidades, actitudes y conocimientos que las personas aplican para relacionarse con los demás, realizar algún trabajo o actividad, resolver un problema o intervenir en su entorno.
} 
la reputación colectiva y la eficiencia del sistema de valor. Estas competencias permiten a las organizaciones aprovechar los recursos y capacidades dentro de cada empresa, así como las redes y la acción colectiva; e incluyen aptitudes especiales para el desarrollo sistemático de procesos y para descubrir nuevas formas de desarrollar estos procesos y fomentar la innovación de productos y procesos.

Este análisis concuerda con el de Marshall: la concentración de empresas en un distrito industrial favorece la división del trabajo y, por lo tanto, la generación de economías externas. "Esta elemental localización de la industria gradualmente preparó el camino para muchos de los modernos desarrollos de la división del trabajo en las artes mecánicas y en la tarea de la administración de empresas" (Marshall, 1890). Las economías externas surgirían, por ejemplo, cuando una determinada industria escogiese alguna localidad para su desarrollo ya que se obtendrían ventajas desde el punto de vista de la disponibilidad de personas con habilidades específicas para el mismo sector productivo; generación y difusión de nuevas ideas, así como desarrollo de actividades subsidiarias en la vecindad, que proveerían con implementos y materiales; aunque en ocasiones podría generarse una demanda excesiva por una misma clase de trabajo. Marshall distinguió las economías derivadas de un incremento en la escala de la producción de cualquier clase de bienes, en dos clases: economías externas como consecuencia de la expansión de toda la industria, y que daría lugar a que las fábricas se congregasen alrededor de ciudades y distritos industriales; y economías internas, que resultarían de los recursos de cada empresa individualmente, de su organización y de la eficiencia de su administración.

Piero Sraffa, en su famoso ensayo de 1926 (Sraffa, 1926), criticaba el hecho de que el punto de vista clásico — que consideraba que se lograrían mayores rendimientos gracias a una mayor división del trabajo interna, hecha posible por el crecimiento de las dimensiones de la firma- había sido totalmente abandonado en la teoría de Marshall, dado que era incompatible con las condiciones de la competencia y obligaba a aceptar que en los mercados se imponían los intereses de las empresas más grandes. Por otro lado, apuntaba Sraffa, cada vez se hacía más énfasis en la importancia de las economías externas, es decir, no de aquellas ventajas que los empresarios obtienen del crecimiento individual, sino del de la industria en su conjunto. La crítica de Sraffa era motivada por una exigencia de realismo cuando señalaba que el concepto de competencia en Marshall era hasta cierto punto complejo y no podía ser reducido a una situación perfectamente competitiva, es decir, en la cual la 
Economía Informa núm. 383 noviembre - diciembre • 2013 | " " "

empresa puede vender sin dificultades todo lo que puede producir y no puede influir sobre el precio, y sin embargo, las numerosas menciones de Marshall a los "mercados especiales" no podían ser consideradas como una teoría acabada de la competencia imperfecta.

Marshall señala que los distritos industriales se caracterizan por ser "concentraciones de sectores especializados en una localidad específica"; en los que las empresas se benefician por el mismo tipo de economías que se encuentra al interior de las grandes empresas, ya que se tienen grandes ventajas por disponer de un mercado de trabajo constante. A partir de sus investigaciones, entre otras, de la industria de cubiertos de Sheffield, propone que, al crecer el distrito, crece también la población de trabajadores capacitados y especializados de los cuales el distrito puede beneficiarse (Marshall, IV.IX.25).

En los distritos industriales italianos, la localización en un área única favorece el desarrollo de proveedores y aumenta la eficiencia a partir de la división social del trabajo, la facilidad de traslado de los individuos de una empresa a otra, y la generación de competencias, además de que promueve la innovación y la difusión del conocimiento entre las pequeñas y medianas empresas del distrito industrial, por el hecho de que empresarios y trabajadores viven en una misma comunidad en la que los "secretos industriales" se encuentran dentro de la atmósfera industrial.

La teoría ortodoxa considera al mercado como un lugar en el que se realizan actividades de intercambio impersonales; donde los agentes compran y venden productos con características bien definidas, a precios tales que equilibran la demanda y la oferta. Para esta teoría, los precios son los principales medios de comunicación entre los agentes, los cuales, al momento de decidir qué acciones estratégicas llevar a cabo, se basan en información que estos precios transmiten. Por esto mismo, las relaciones entre los agentes no son el aspecto más relevante en el mercado, sino el valor que cada agente, individualmente, atribuye a cada bien intercambiado en el mercado; valores que el mercado transforma en los precios de tales bienes.

Una cuestión relevante es la razón que lleva a la formación de los distritos. Pueden ser las economías que se obtienen de vínculos cercanos entre las empresas, o bien los beneficios de la co-localización, los "spillovers" o la necesidad del contacto personal para la transmisión del conocimiento. Desde algunas teorías del desarrollo, se han empleado los conceptos de "capital humano" o de "capital social", en los que la gente es la fuerza detrás del crecimiento regional. En el ámbito no privado, por ejemplo, están los estudios de Robert Putnam 
(1993), quien construyó un índice — “Índice de Comunidad Cívica”10 — para medir el nivel de desarrollo social de diversas regiones en Italia, relacionándolo con el desempeño económico de cada una. Concluye que la causa de los distintos desempeños económicos se explicaba por la diferente calidad de la "comunidad cívica". ${ }^{11}$ El desempeño del distrito industrial dependerá del capital social constituido por estas economías (externas, de acuerdo con la definición de Marshall) y competencias. Los lugares con vínculos densos y altos niveles de capital social tradicional ofrecen ventajas a los miembros $y$, de esta forma, promueven la estabilidad, en cambio los lugares con vínculos débiles y redes menos densas están abiertos a la entrada y, de esta forma, promueven nuevas combinaciones de recursos e ideas. Algunos critican el concepto de capital social porque, en última instancia, está referido a un tejido social conservador que contrasta con las aspiraciones de las personas creativas, que buscan una mayor libertad en todos los ámbitos.

Por su parte, Grandinetti y Tabacco señalan que los distritos se han convertido en "meta-contextos con una alta densidad de sitios de producción de conocimiento (contextos de la firma) y una alta densidad de canales de transferencia de conocimiento que, a su vez, alimentan los procesos de creación de conocimiento" (Grandinetti y Tabacco, 2003). La interacción entre las empresas permite difundir en el tejido social (y, en primer lugar, entre los empresarios) informaciones sobre las tecnologías, los materiales, los productos: produce, en otras palabras, informaciones económicamente relevantes sobre posibles oportunidades de beneficio y la información que deriva de la experiencia directa de otros empresarios se transmite más rápidamente en el distrito industrial que en otros modelos de manufactura, induciendo un efecto de verdadero "contagio informativo" (Brusco, Minerva, Poli, Solinas, julio, 2001; Grandinetti e Tabacco, 2003; Camisón, César, 2003).

\footnotetext{
${ }^{10}$ Para construir su Índice de Comunidad Cívica. Putnam reunió cuatro indicadores: el número de asociaciones por habitante, deportivas (la gran mayoría), de recreación, científicas, culturales, técnicas, económicas, de salud, de servicio social, etc.; la lectura de periódicos, que muestra el interés de las personas por los asuntos públicos; la participación en referéndum, que no estaban distorsionados por el fenómeno del clientelismo en las regiones del Sur; el voto de preferencia por un candidato particular, opción "voluntaria" que en los hechos era resultado de prácticas clientelares y que se utilizó, por lo tanto, como indicador de ausencia de comunidad cívica.

${ }^{11}$ Según la definición que da Putnam, la comunidad cívica comprende algunos aspectos esenciales como el compromiso cívico, igualdad política, solidaridad, confianza y tolerancia entre los ciudadanos, lo que no implica la desaparición del conflicto; y la existencia de asociaciones civiles. El capital social consiste en un conjunto de relaciones sociales de las cuales disponen los sujetos - empresarios, trabajadores, entes gubernamentales, etc. - en un determinado momento y que les permite promover la adquisición de conocimientos y el logro de objetivos que, de otra forma, sería sumamente costoso alcanzar.
} 
Economía Informa núm. 383 noviembre - diciembre • 2013 | " " "

Becattini (2004), a su vez, señala que "han sido necesarios muchos años para que se apreciara, por un lado, la insustituibilidad y el valor económico de la transmisión cara a cara del know how productivo; y del otro, el papel y el valor de instituciones informales, como las costumbres comerciales, en el desarrollo de los intercambios". Otra explicación (Florida, 2003), es que las empresas se aglomeran (cluster) para obtener ventajas de la concentración de gente con talento, que potencia la innovación y el crecimiento económico.

De acuerdo con la teoría ortodoxa, los lugares crecen ya sea porque están ubicados en rutas de transporte o porque están dotados de recursos naturales que atraen a las firmas para que inviertan en ellos. La importancia económica de un lugar está ligada a la eficiencia con la que se hacen los negocios y los gobiernos se basan en esta teoría para justificar las exenciones fiscales y los bajos salarios para atraer inversiones.

El distrito es una comunidad que comparte, no solamente un territorio, sino una historia, una cultura, tradiciones y un conjunto de valores y normas de convivencia. Es en este ambiente social, político y cultural que se generan, de manera colectiva, un conjunto de competencias que comparten las empresas al interior del distrito. El distrito es, por lo tanto, una comunidad cognitiva definida por el sentido de pertenencia de los miembros, ya que el sentir que se pertenece implica asumir como propios la historia, la cultura, tradiciones y, de manera fundamental, los valores y normas de convivencia. Sólo a partir de la aceptación de estos principios y normas puede generarse la confianza mutua necesaria para ejercer una administración estratégica en conjunto en la que prevalezcan la competencia y la colaboración entre empresas.

Cuando se habla de la nueva economía de redes y de modalidades novedosas de organización industrial — como es el caso de los distritos industriales - es necesario hacer énfasis en el papel fundamental que desempeña el conocimiento, dado que se trata de "comunidades cognitivas" .Desde una perspectiva basada en el conocimiento, dado que el conocimiento generado o adquirido y las capacidades con las que se cuenta en una organización son únicos y difíciles de imitar, confieren una ventaja competitiva sustentable. La medida en la cual la empresa efectivamente sea exitosa depende de qué tan efectiva sea en el momento de combinar el conocimiento y las competencias en configuraciones que le otorguen valor a su mercado o su comunidadcompetencias distintivas individuales. El conocimiento, en una visión de la empresa basada en el conocimiento, es acumulativo y sus rendimientos crecientes; cuanto más la empresa sepa, más podrá aplicar aquello que sabe a nuevas áreas de oportunidad. 
De acuerdo con Méndez, a mediados de la década de los 1990, las aportaciones sobre medios o ambientes innovadores (milieux innovateurs) o sobre las ventajas competitivas de naciones o regiones, supusieron una renovación en relación con las visiones individualistas neoschumpeterianas. Para aquellas, la innovación en las empresas resulta, en gran medida, de un entorno territorial (social, económico, cultural) con características específicas; por esta razón, "un esfuerzo sostenido en la generación y difusión de la innovación resulta hoy un factor clave, tanto para mejorar la competitividad de las empresas, como para favorecer un desarrollo los territorios, no solo en términos de crecimiento económico, sino desde una perspectiva integrada y atenta a la calidad de vida de que disfruta su población” (Méndez, 2006).

En la medida en que los distritos están basados en la interconexión y organización en red de un conjunto de empresas, la toma de decisiones se realiza de una manera no individualista y la información para la toma de decisiones es compartida al interior del distrito. Esto constituye una experiencia de administración estratégica que podríamos denominar "compartida". De hecho, dadas las condiciones de incertidumbre en las que los agentes no pueden prever todas las consecuencias de sus acciones, la toma de decisiones por parte del empresario del distrito se basa, más que en prever exactamente cuáles consecuencias resultarán de las interacciones en las que participará, en decidir con quién interactuar.

Lester y Piore señalan que los distritos industriales son comunidades interpretativas, dado que el conocimiento y entendimiento que circulan de una empresa a otra tienen las propiedades de un lenguaje y evolucionan a través de la conversación (Lester \& Piore, 2004). Según estos autores, los sPLs han desarrollado una conciencia de sí mismos y han creado estructuras internas de administración con el objeto de conservar y enriquecer el proceso de conversación, así como de orientarlo estratégicamente. Se trata de un ambiente con determinados patrones de organización social cuyos elementos principales serían un sentimiento de pertenencia, un concepto de arraigamiento ("rootedness" o "embeddedness"), la existencia de una comunidad cognitiva y de un conjunto de instituciones y reglas paralelas al sistema de valores que posibilitan la diseminación de estos valores dentro de la red local, los sostienen y transmiten entre generaciones y permiten regular el sistema (Russo y Ruini, 2004).

La competencia que prevalece en el ámbito de los SPL, en particular en la forma de distrito industrial, tiene poco que ver con la competencia perfecta delineada por Marshall, sin embargo, los conceptos que surgen de sus estudios empíricos, a diferencia de la teoría del equilibrio parcial, proporcionan una 
Economía Informa núm. 383 noviembre - diciembre • 2013 | " " "

base útil para estudiar aspectos como la generación y difusión del conocimiento en ambientes industriales. De hecho, en Industry and Trade, Marshall explica que el concepto de costo de producción que propone en Principles no tiene nada qué ver con el mundo real.

\section{Conclusión}

El desarrollo de los distritos industriales responde a un proceso en el que ha entrado en crisis la producción en serie y a grandes escalas de productos indiferenciados de la época fordista. Como resultado de este proceso, las ventajas competitivas más importantes para las empresas radican, fundamentalmente, en la capacidad de generar conocimientos y competencias que se constituyan en ventajas competitivas. Estas ventajas no son de fácil acceso y, al menos en el caso del conocimiento y la información, implican altos costos a los que las pequeñas y medianas empresas individualmente no siempre pueden hacer frente.

Algunos de los conceptos más importantes, cuando se habla de distritos industriales, los propuso Marshall: la "atmósfera industrial" — concepto ambiguo y de difícil concreción-y las “economías externas". Ambos conceptos, al igual que el otro, evasivo y difícil de aprehender — la "entrepreneurship"-, se refieren a cualidades externas — un ambiente creado por los integrantes del distrito gracias a su interacción y cualidades internas- que reflejan un cierto desarrollo social, cultural y político.

El estudio de los distritos industriales y del papel que han jugado en la generación y desarrollo del conocimiento presenta oportunidades para instrumentar estrategias de promoción para las pequeñas empresas en otros ámbitos. En el caso de México, resulta enriquecedora la experiencia de los distritos industriales italianos dadas algunas similitudes, como la existencia de sistemas productivos locales tanto de producción industrial como de tradiciones artesanales y conocimientos locales con hondas raíces. El potenciamiento de conocimientos locales puede resultar también importante para desarrollar localidades desde una perspectiva de aprovechamiento integral y sostenible del medio ambiente. 




\section{Bibliografía}

Becattini, Giacomo (2004), Vicisitudes y potencialidades de un concepto: el distrito industrial. Universidad Internacional Menéndez Pelayo, Barcelona. Centre Ernest Lluch.

Brenner, T. (2000), Industrial districts: a typology from an evolutionary perspective, MaxPlanck-Institute, Munchen

Camisón, C. y March, I (1995), “Estrategias de cooperación y de innovación con soporte territorial y parques tecnológicos: un estudio empírico". Revista Europea de Dirección y Economía de la Empresa, 4 (1), pp. 11-33.

Camisón, César (2003), "Shared, Competitive and Comparative Advantages: A Competence-Based View of the Competitiveness of Industrial Districts". Trabajo presentado en la Conference on Clusters, Industrial Districts and Firms: the Challenge of Globalization Conference in Honour of Professor Sebastiano Brusco; Módena, Italia. Septiembre 12-13, 2003.

Casas, Rosalba y Jorge Dettmer (2007), Sociedad del conocimiento, capital intelectual y organizaciones innovadoras. IIS-UNAM.

Cimoli Mario (2000), ed. Developing Innovation System. México in a Global Context, GB, EUA: Continuum.

Jasso, Javier, y Arturo Torres (1997), “Aprendizaje tecnológico y desempeño competitivo en las empresas de autopartes y petroquímicas mexicanas”, Documento de Trabajo, No. 70 cide, México D.F.

Jasso, Javier y Arturo Torres (2008), "Innovación y gestión del conocimiento: debate y perspectiva empresarial en el mundo global". en Micheli J., E. Medellín, A. Hidalgo y J. Jasso (coordinadores) Conocimiento e Innovación: Retos de la empresa en la sociedad del conocimiento. Plaza y Valdés, UAm, FCA-UnAm-ALTEC

Lane, David, "Distretti industriali come sistemi complessi". http://www.davidlane.eu/ unimore. 2003

Marshall, Alfred (1890), Principles of Economics. $8^{\circ}$ Edición (Londres: MacMillan; 1920)

Marshall, Alfred (1919), Industry and Trade. $3^{\circ}$ Edición (Londres: MacMillan; 1920) Markusen, Ann (1996), "Sticky Places in Slippery Space: A Typology of Industrial District”, in Economic Geography, Vol. 72.

Polanyi, M (1966), The Tacit Dimension. New York: Anchor Day.

Porter, Michael (1987), Ventaja Competitiva. México: CECSA

- (1991), La Ventaja competitiva de las naciones. España: Plaza \& Janés Editores, S.A.

(1998), On Competition, Harvard Business School Press, Boston 
Economía Informa núm. 383 noviembre - diciembre • 2013

Putnam, Robert D. (1993), “The Prosperous Community”. The American Prospect. vol. 4, nr. 13, marzo 21, 1993.

Russo, Margherita (1997), "Relazioni tra Imprese e Sviluppo Locale". Economia e Politica Industriale, 93, pp. 105-137.

Russo, Margherita, Fabio Ruini (2004), Analisi del processo di innovazione all'interno del distretto ceramico di Sassuolo-Scandiano. www.fabioruini.eu/unimore UnesCo (2005), Hacia las sociedades del conocimiento. 